\section{Quantized thermal transport in single-atom junctions}

\author{
Longji Cui, ${ }^{1 *}$ Wonho Jeong, ${ }^{1 *}$ Sunghoon Hur, ${ }^{1}$ Manuel Matt, ${ }^{2}$ Jan C. Klöckner, ${ }^{2}$ \\ Fabian Pauly, ${ }^{2}$ Peter Nielaba, ${ }^{2}$ Juan Carlos Cuevas, ${ }^{3} \dagger$ \\ Edgar Meyhofer, ${ }^{1} \dagger$ Pramod Reddy ${ }^{1,4} \dagger$
}

Thermal transport in individual atomic junctions and chains is of great fundamental interest because of the distinctive quantum effects expected to arise in them. By using novel, custom-fabricated, picowatt-resolution calorimetric scanning probes, we measured the thermal conductance of gold and platinum metallic wires down to singleatom junctions. Our work reveals that the thermal conductance of gold single-atom junctions is quantized at room temperature and shows that the Wiedemann-Franz law relating thermal and electrical conductance is satisfied even in single-atom contacts. Furthermore, we quantitatively explain our experimental results within the Landauer framework for quantum thermal transport. The experimental techniques reported here will enable thermal transport studies in atomic and molecular chains, which will be key to investigating numerous fundamental issues that thus far have remained experimentally inaccessible.

I he study of thermal transport at the nanoscale is of critical importance for the development of novel nanoelectronic devices and holds promise to unravel quantum phenomena that have no classical analogs (1-3). In the context of nanoscale devices, metallic atomic-size contacts (4) and single-molecule junctions (5) represent the ultimate limit of miniaturization and have emerged as paradigmatic systems revealing previously unknown quantum effects related to charge and energy transport. For instance, transport properties of atomic-scale systems-such as electrical conductance $(6)$, shot noise $(7,8)$, thermopower (9-11), and Joule heating (12)-are completely dominated by quantum effects, even at room temperature. Therefore, they drastically differ from those of macroscale devices. Unfortunately, the experimental study of thermal transport in these systems constitutes a formidable challenge and has remained elusive to date, in spite of its fundamental interest (13).

Probing thermal transport in junctions of atomic dimensions is crucial for understanding the ultimate quantum limits of energy transport. These limits have been explored in a variety of microdevices (14-18), where it has been shown that, irrespective of the nature of the carriers (phonons, photons, or electrons), heat is ultimately transported via discrete channels. The maximum

${ }^{1}$ Department of Mechanical Engineering, University of Michigan, Ann Arbor, Ml 48109, USA. ${ }^{2}$ Department of Physics, University of Konstanz, D-78457 Konstanz, Germany. ${ }^{3}$ Departamento de Física Teórica de la Materia Condensada and Condensed Matter Physics Center (IFIMAC), Universidad Autónoma de Madrid, Madrid 28049, Spain. ${ }^{4}$ Department of Materials Science and Engineering, University of Michigan, Ann Arbor, MI 48109, USA. *These authors contributed equally to this work. fCorresponding author. Email: juancarlos.cuevas@uam.es (J.C.C.); meyhofer@ umich.edu (E.M.); pramodr@umich.edu (P.R.) contribution per channel to the thermal conductance is equal to the universal thermal conductance quantum $G_{0, T h}=\pi^{2} k_{\mathrm{B}}^{2} T / 3 h$, where $k_{\mathrm{B}}$ is the Boltzmann constant, $T$ is the absolute temperature, and $h$ is the Planck's constant. However, observations of quantum thermal transport in microscale devices have only been possible at sub-Kelvin temperatures, and other attempts at higher-temperature regimes have yielded inconclusive results (19).

The energy-level spacing in metallic contacts of atomic size is of the order of electron volts (i.e., much larger than thermal energy); therefore, these junctions offer an opportunity to explore whether thermal transport can still be quantized at room temperature. However, probing thermal transport in atomic junctions is challenging because of the technical obstacles in reproducibly creating stable atomic junctions while measuring the miniscule heat currents flowing through the atomic chains. We present an experimental platform that allows us to measure the thermal conductance of metallic wires down to the single-atom limit. With this technique, we were able to observe quantized thermal transport at room temperature. Specifically, we developed an approach that uses custom-fabricated calorimetric scanning thermal microscopy (C-SThM; Fig. 1) probes, which feature a very large thermal resistance $\left(R_{\mathrm{P}} \approx 1.3 \times 10^{6} \mathrm{~K} / \mathrm{W}\right)$ and high-resolution Pt thermometers with a temperature resolution $\left(\Delta T_{\min }\right)$ of $\sim 0.6 \mathrm{mK}$ in a $10-\mathrm{Hz}$ bandwidth (20-22). These characteristics enable thermal conductance measurements with $\sim 25-\mathrm{pW} / \mathrm{K}$ resolution when a temperature bias of $\sim 20 \mathrm{~K}$ is applied across atomic junctions. We achieved the large thermal resistance by incorporating long, T-shaped beams with a small cross-sectional area, which also enable a very high stiffness $\left(>10^{4} \mathrm{~N} / \mathrm{m}\right.$ in the normal direction) (20). Both of these fea- tures are critical for accomplishing atomic junction thermal measurements. The C-SThM probes also have a sharp metallic tip that we coated with $\mathrm{Au}$ or Pt, but which can also be coated with other metals (20).

Our strategy for quantitatively measuring the thermal conductance of atomic junctions is depicted in Fig. 1A. We heated the Au substrate to a temperature $T_{\mathrm{S}}=315 \mathrm{~K}$ while the probe was connected to a thermal reservoir at $T_{0}=295 \mathrm{~K}$. We then displaced the probe toward the heated substrate by piezoelectric actuation until we reached an electrical conductance of $4 G_{0}$, with $G_{0}=2 e^{2} / h \approx(12.9 \text { kiloohm })^{-1}$ being the electrical conductance quantum. This electrical conductance signals the formation of a $\mathrm{Au}-\mathrm{Au}$ contact involving several atoms, as established by past work (4). We measured the probe-substrate electrical conductance by applying a small sinusoidal voltage bias with an amplitude of $1 \mathrm{mV}$ at a frequency of $10 \mathrm{kHz}$ and recording the amplitude of the resultant electric current with a lock-in amplifier (20). Once we reached the threshold conductance, we withdrew the probe slowly from the substrate at a rate of $0.05 \mathrm{~nm} / \mathrm{s}$. During this process, the probe-substrate contact region is expected to become more constricted until it forms a single-atom-wide junction, which is broken upon further withdrawal (Fig. 1B) (23). We concurrently performed thermal conductance measurements by continuously measuring the change in probe temperature $\left(\Delta T_{\mathrm{P}}\right)$ in response to heat flow $(Q)$ through the atomic junction. We measured the probe temperature by monitoring the change in the resistance of the embedded Pt resistance thermometer via a sinusoidal electrical current of fixed amplitude $(10 \mu \mathrm{A})$ and frequency $(1 \mathrm{kHz})$ supplied to the probe, while measuring the voltage drop across the resistor.

From the resistance network (Fig. 1A), we directly related the thermal conductance $\left(G_{\mathrm{Th}}\right)$ of the atomic junctions to $\Delta T_{\mathrm{P}}$ by $G_{\mathrm{Th}}=\Delta T_{\mathrm{P}} /\left[R_{\mathrm{P}}\left(T_{\mathrm{S}}-T_{0}-\Delta T_{\mathrm{P}}\right)\right]$, where $R_{\mathrm{P}}$ was $1.3 \times 10^{6} \mathrm{~K} / \mathrm{W}(20)$. Representative thermal (red) and electrical conductance (blue) traces show that the electrical conductance decreases in discrete steps (Fig. 2A). Many of the curves exhibit preferential conductance values that occur at integer multiples of $G_{0}$, as expected from past work (4). The corresponding thermal conductance curves closely correlate to the electrical conductance curves. Further, several of the thermal conductance curves show steps with preferential conductances at $2 \pi^{2} k_{\mathrm{B}}^{2} T / 3 h$, a value that is twice the quantum of thermal conductance $\left(G_{0, T h}\right)$. The factor of 2 is a consequence of the spin degeneracy in electron transport and is not present in the usual definition of $G_{0, T h}$ because it was introduced in the context of phonon transport, where spin degeneracy is absent.

Past studies (24-26) on electrical conductance quantization established that the presence of plateaus in the conductance traces is insufficient evidence of quantization. Definitive conductance quantization requires an unbiased statistical analysis from a large data set. We obtained $\sim 2000$ consecutive electrical and thermal conductance traces with the procedure outlined above, which 
Fig. 1. Experimental setup and scanning electron microscopy (SEM) images of the scanning thermal probes. (A) Schematic of a calorimetric scanning thermal microscopy (C-SThM) probe, which is used to make atomic junctions with a heated metallic substrate. The tip and substrate coatings can be either Au or Pt. The electrical conductance of the tip-substrate junction is monitored by applying a small sinusoidal voltage bias and measuring the resultant current. The resistance of the Pt resistance thermometer is monitored by applying a sinusoidal current (amplitude $I_{f}$ ) and recording the voltage output $\left(V_{f}\right)$. A thermal resistance network describes the resistances of the atomic chain $\left[R_{\text {Atom }}=\left(G_{\mathrm{Th}}\right)^{-1}\right]$ and the scanning probe $\left(R_{\mathrm{P}}\right)$, as well as the heat current flow ( $Q$, red arrow). The substrate, thermal reservoir, and tip temperatures are indicated by $T_{\mathrm{S}}, T_{\mathrm{O}}$, and $T_{\mathrm{P}}$, respectively. (B) Schematics of the atomic chains forming, narrowing, and breaking during the withdrawal of the probe from the heated substrate. (C) SEM image (side view) of a scanning probe with two long and stiff T-shaped $\mathrm{SiN}_{x}$ beams. (D) SEM image (top view) of the scanning probe, featuring a Au-coated tip (inset) and a serpentine Pt thermometer.

Fig. 2. Thermal conductance quantization in Au atomic junctions. (A) Representative traces of thermal and electrical conductances measured while reducing the transverse constriction of $\mathrm{Au}$ atomic junctions by displacing the Au tip of the C-SThM probe away from the Au substrate. The thermal (red) and electrical (blue) conductance traces are plotted in units of $2 \pi^{2} k_{B}^{2} T / 3 h\left(2 G_{0, T h}\right.$, twice the thermal conductance quantum) and $2 e^{2} / h\left(G_{0}\right.$, the electrical conductance quantum), respectively. (B) Histogram of the ratio of the thermal conductance to the electrical conductance, showing good agreement with the Lorenz number $\left(L / L_{0}\right)$. The color bar indicates the number of counts, increasing from white to red. (C and D) Electrical and thermal conductance histograms obtained from 2000 concurrently measured electrical and thermal conductance traces (without any data selection), similar to those shown in (A). (E) A joint plot of the electrical and thermal conductance traces shows the tight correlation between the occurrence of electrical and thermal conductance quantization. (F) Analysis of the data in (C) and (D) shows that the WiedemannFranz law accurately predicts the thermal conductance of Au atomic junctions (the peak is at 1.06). a.u., arbitrary units.
A

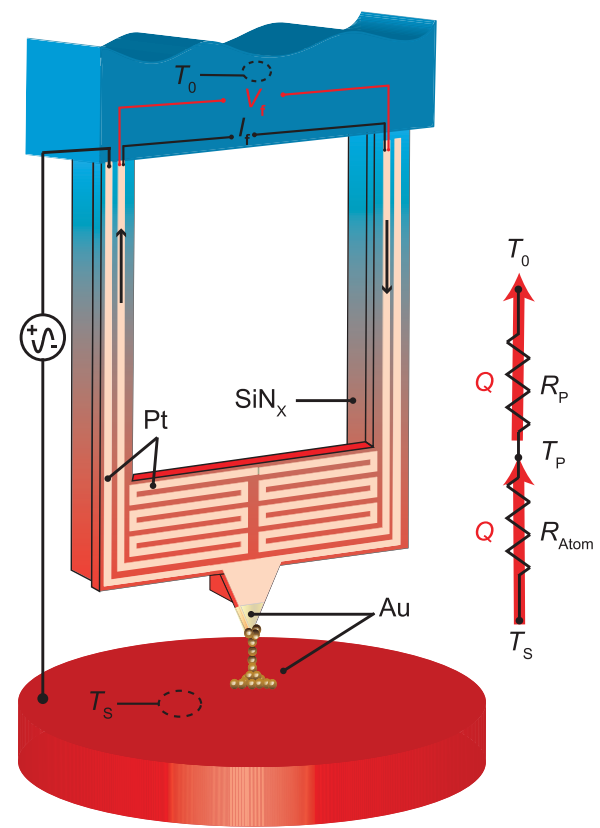

B

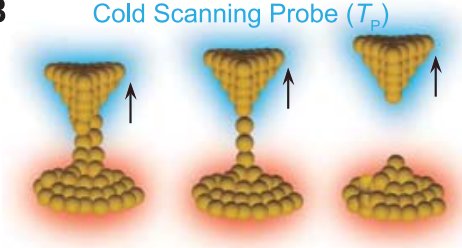

Hot Substrate $\left(T_{\mathrm{s}}\right)$

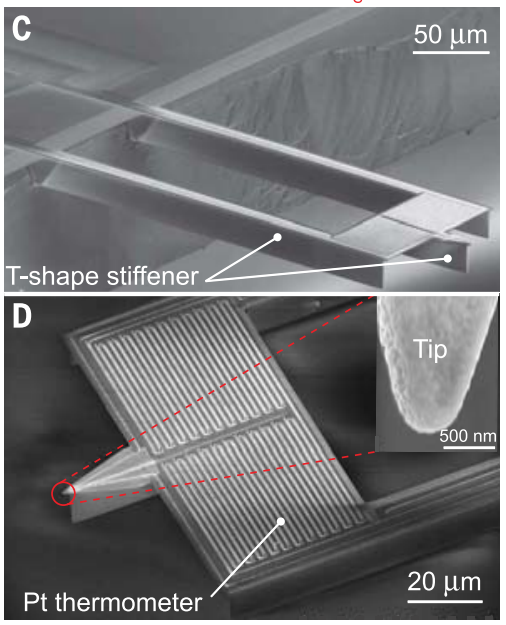

$\mathbf{A}_{\widehat{\mathcal{1}}}$

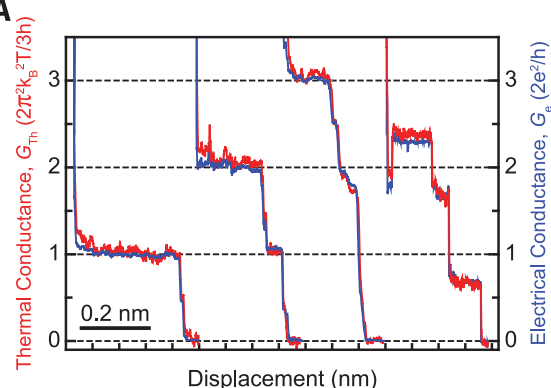

C

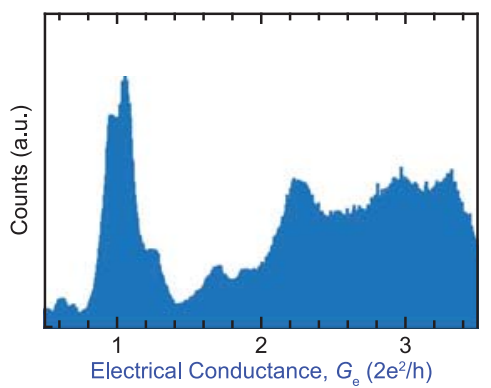

E

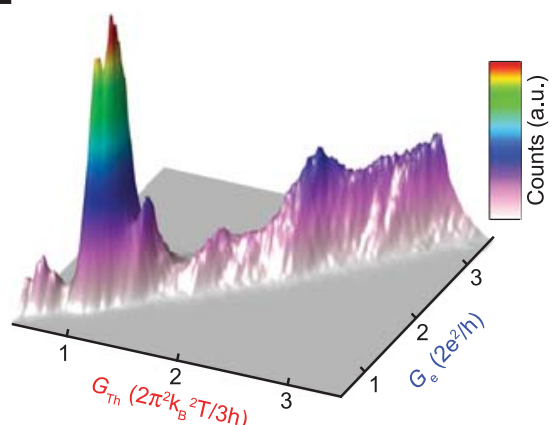

B

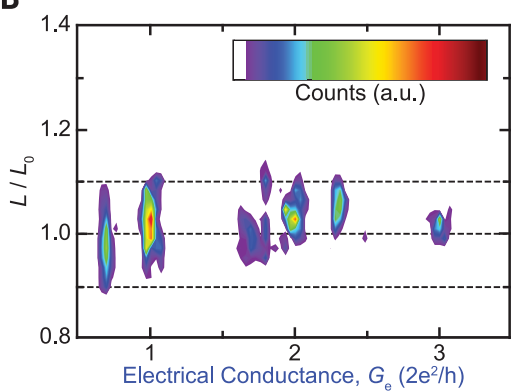

D

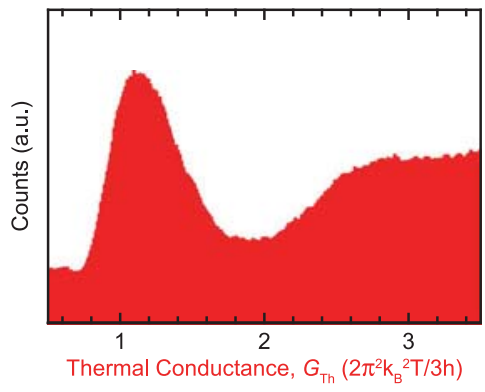

$\mathbf{F}$

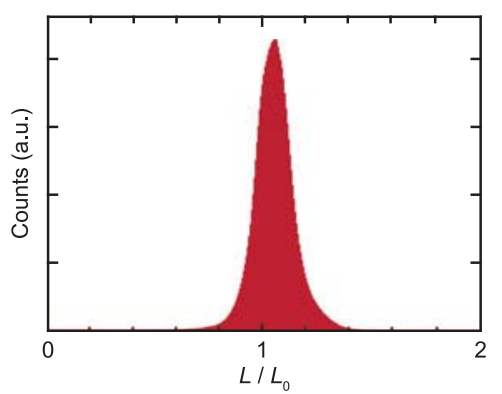


A

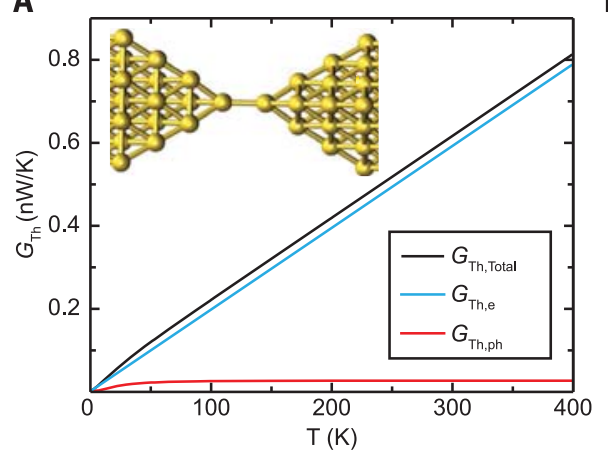

C
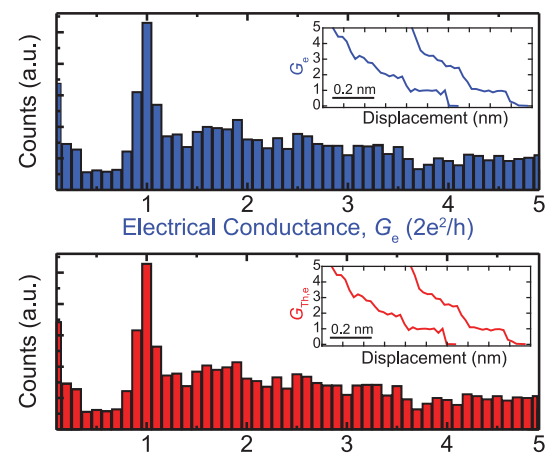

Electronic Thermal Conductance, $G_{T h, e}\left(2 \pi^{2} k_{B}{ }^{2} T / 3 h\right)$
B

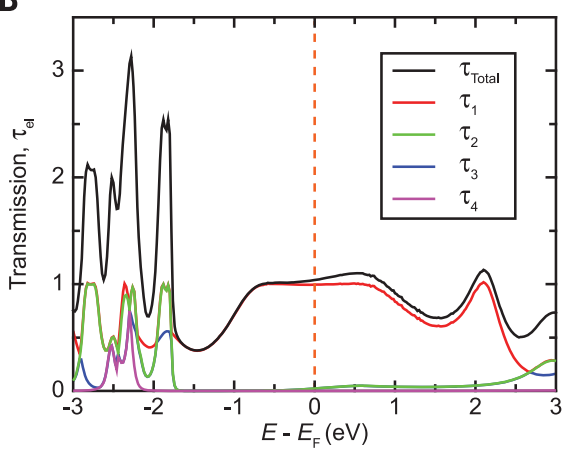

D

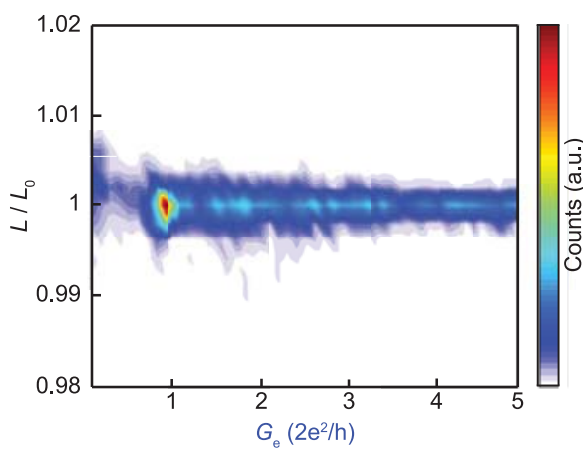

Fig. 3. Computations of thermal conductance in Au atomic junctions. (A) Computed thermal conductance as a function of temperature for the Au atomic junction geometry shown in the inset. The total thermal conductance $G_{\text {Th,total }}$ (black), the electronic contribution $G_{\text {Th,e }}$ (blue), and the phononic contribution $G_{\text {Th,ph }}$ (red) to thermal conductance are shown. $G_{T h, p h}$ is almost negligible at room temperature. (B) The total electronic transmission $\tau_{\text {total }}$ and the individual electronic transmission coefficients $\tau_{1}$ to $\tau_{4}$, as a function of the energy (measured with respect to the Fermi energy $E_{F}$ ) for the contact geometry in the inset of $(A)$. The electronic transmission is dominated by a single (spin-degenerate) conduction channel. (C) Electrical and electronic thermal conductance histograms obtained from 100 MD simulations of the formation of $\mathrm{Au}$ atomic contacts at room temperature (insets show representative electrical and thermal conductance traces obtained from these simulations). (D) Lorenz ratio $\left(L / L_{0}\right)$ as a function of the electrical conductance $G_{e}$ of the Au contacts, as obtained from the histograms in (C). The ratio is very close to 1 . Deviations are below $1 \%$, which shows that the Wiedemann-Franz law is fulfilled with good accuracy, irrespective of the contact size.

yielded electrical and thermal conductance histograms with clear peaks at $1 G_{0}$ and $2 G_{0, T h}$, respectively (Fig. 2, C and D). The thermal conductance histogram is broader than its electrical counterpart because the time constant for our thermal measurements $(\sim 25 \mathrm{~ms})(20)$ is larger than that for our electrical measurements $(\sim 10 \mu \mathrm{s})$.

The close correlation between the thermal and electrical conductances (Fig. 2A) provides important information regarding the validity of the Wiedemann-Franz law, which relates the electronic contribution of the thermal conductance, $G_{\mathrm{Th}, \mathrm{e}}$, to the electrical conductance, $G_{\mathrm{e}}$, by $G_{\mathrm{Th}, \mathrm{e}}=L_{0} T G_{\mathrm{e}}$, where $L_{0}=\left(\pi^{2} / 3\right)\left(k_{\mathrm{B}} / e\right)^{2}$ is the Lorenz number (27). Because this basic law was originally derived with semiclassical arguments and is approximately obeyed in macroscopic wires of standard metals (27), it is unclear whether it should remain valid in metallic atomic junctions, where the transport mechanisms are different (28). To test the validity of the Wiedemann-Franz law in atomic junctions, we used the data in Fig. 2A to obtain the Lorenz ratio, $L / L_{0}=G_{\mathrm{Th}} / L_{0} G_{\mathrm{e}} T$, as a function of $G_{e}$. Here, $T=305 \mathrm{~K}$ is the av- erage temperature of the tip and the sample. This process was repeated for each set of curves shown in Fig. $2 \mathrm{~A}$, and the data from all four curves were collected into the two-dimensional (2D) histogram shown in Fig. 2B (20). It is clear from the data that the value of $L / L_{0}$ is very close to 1 .

We plotted a 3D histogram from 2000 concurrently measured electrical and thermal conductance traces, without any data selection (Fig. 2E) (20). The histogram features a large peak corresponding to $\left(G_{0}, 2 G_{0, T h}\right)$, showing that the electrical and thermal conductance quantization occurs concurrently and that the quantized state is a statistically favored atomic configuration. This close correlation is also reflected in the $2 \mathrm{D}$ histogram (Fig. 2F), where we computed $L / L_{0}=G_{\mathrm{Th}} / L_{0} G_{\mathrm{e}} T=$ 1.06 as mean value from the 2000 traces (20). The small increase above 1.00 is primarily due to contributions from phonons to the thermal conductance, which we estimate to add $\sim 5$ to $10 \%$ (see below). The increase in $L / L_{0}$ could also reflect small contributions ( 10 to $20 \mathrm{pW} / \mathrm{K})$ from nearfield radiative heat transfer (29) and even smaller contributions from the overestimation of the ther- mal conductance during periods of rapid transition in the electrical conductance, when the thermal response lags the electrical response.

To unambiguously identify the origin of our quantization observations, we used customdeveloped methods to compute the thermal and electrical conductance of Au atomic-size contacts within the framework of the Landauer-Büttiker formalism for coherent transport. We exactly simulated the experiments by using a combination of molecular dynamics (MD) $(11,30,31)$, density functional theory (DFT) $(32,33)$, and nonequilibrium Green's function techniques to describe the contributions of both electrons and phonons to the thermal conductance (20). Our MD simulations (movies S1 to $\mathrm{S} 6$ ) show that when the electrical conductance is $G_{0}$, the atomic junction typically has an atomic "dimer" bridge geometry, similar to that shown in the inset of Fig. 3A. Our DFT-based calculations (Fig. 3A) for the electrical and thermal transport for a dimer geometry show that the thermal conductance at room temperature is dominated by the electronic contribution, with phonons giving only $\sim 4 \%$ of the total signal, which is similar to the case of bulk Au wires (34). We also found that the electronic contribution at room temperature in this example is $G_{\mathrm{Th}, \mathrm{e}} \approx 0.59 \mathrm{nW} / \mathrm{K}$, which is close to twice the thermal conductance quantum. The reason for this quantized thermal conductance is apparent from Fig. 3B, where we show that electronic transmission is dominated by a single (spin-degenerate) conduction channel and has a value very close to 1 . This fact, taken together with the smooth energy dependence of the electronic transmission around the Fermi energy (20), leads to excellent agreement with the Wiedemann-Franz law: $G_{\mathrm{Th}, \mathrm{e}} \approx L_{0} T G_{\mathrm{e}} \approx 2 G_{\mathrm{O} \text {,Th }}$. Therefore, we conclude that for one-atom $\mathrm{Au}$ junctions, the observed thermal conductance quantization is intricately linked to the electronic structure of this monovalent metal.

Having established the origin of thermal conductance quantization and the small contribution of phonons, we focused on the analysis of $G_{\text {Th,e }}$ to systematically study the validity of the Wiedemann-Franz law for Au contacts of arbitrary size. For this purpose, we performed 100 simulations of the formation of Au junctions at $300 \mathrm{~K}$ while computing the electrical and thermal conductance for the transient geometries. We present histograms constructed from the electrical and thermal conductance traces (Fig. 3C). The electrical conductance histogram is dominated by a peak close to $1 G_{0}$, which is due to preferential formation of one-atom-thick contacts and short atomic chains (11). The corresponding histogram for the thermal conductance (normalized by $2 G_{0, \mathrm{Th}}$ ) shows a very close correlation with the electrical one. Therefore, the Wiedemann-Franz law, $G_{\mathrm{Th}, \mathrm{e}} \approx L_{0} T G_{\mathrm{e}}$, holds almost exactly, irrespective of the electrical conductance value (i.e., contact size) (Fig. 3D). Although some fine details of the experimental histograms are not reproduced, these computational results are in excellent agreement with our experiments (Fig. 2) and provide compelling evidence that the quantized thermal 
A

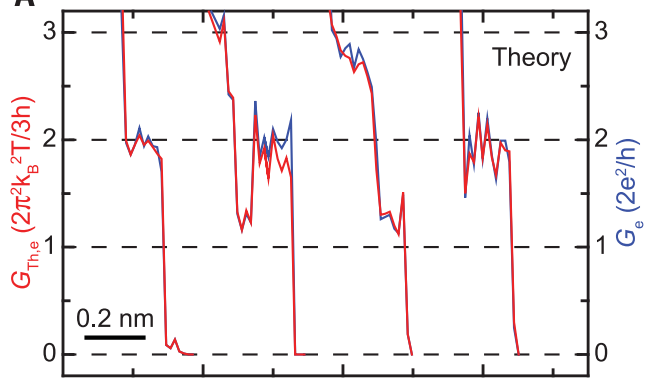

Displacement $(\mathrm{nm})$

C

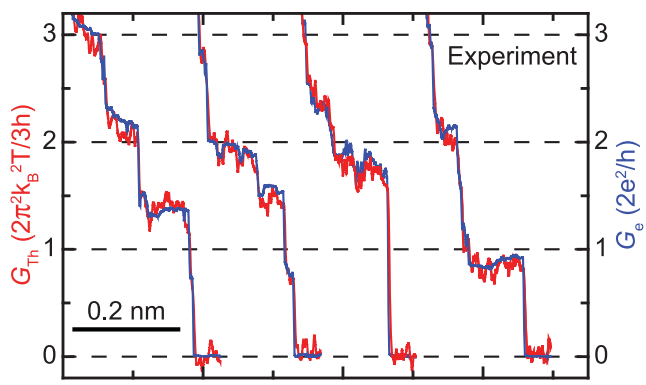

Displacement $(\mathrm{nm})$

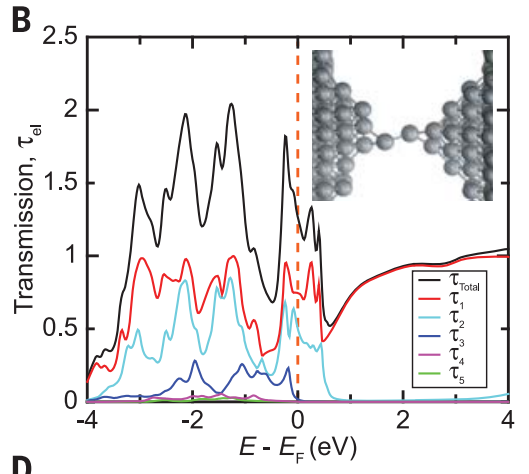

D

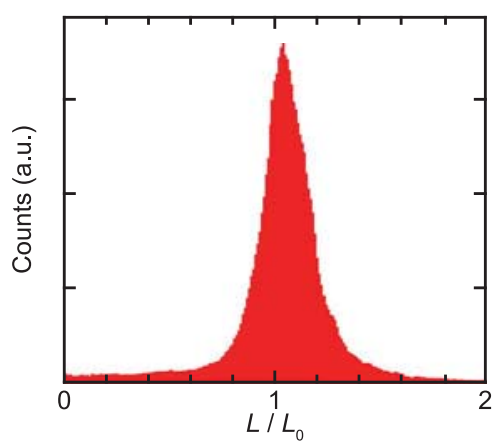

Fig. 4. Calculated and measured transport properties of $\mathrm{Pt}$ atomic junctions. (A) Representative traces of the electrical conductance (blue) and the electronic thermal conductance (red) for Pt junctions, calculated by combined MD and transport simulations. (B) The total electronic transmission and the individual electronic transmission coefficients as a function of the energy (measured with respect to the Fermi energy $E_{\mathrm{F}}$ ) for the contact geometry shown in the inset. The electronic transmission has substantive contributions from three channels at the Fermi level. In addition, the transmission varies more rapidly with energy than for Au atomic junctions. (C) Representative measured traces of electrical (blue) and thermal (red) conductances for Pt atomic junctions show discrete steps (conductance histograms do not display electrical or thermal conductance quantization) (20). (D) Histogram similar to that shown in Fig. 2F but for Pt data, showing that the Wiedemann-Franz law is applicable (the peak is at 1.04). The corresponding theoretical results can be seen in fig. S10 (20).

transport that we observed corresponds to $\mathrm{Au}$ single-atom junctions and is not affected by surface contaminants, which are known to plague nano-gap thermal transport measurements (29).

The observed thermal conductance quantization in $\mathrm{Au}$ junctions is a direct consequence of the electronic structure of these junctions, which in turn is also responsible for the validity of the Wiedemann-Franz law. However, the opposite is not true: The validity of the Wiedemann-Franz law is a consequence of the smooth energy dependence of the electronic transmission $(35,36)$, but this does not imply that thermal transport is quantized in all cases. For example, our simulations of the Pt atomic junctions suggest that although the electrical and thermal conductance traces show discrete steps and are in agreement with the Wiedemann-Franz law (Fig. 4A), one should not expect electrical or thermal conductance quantization (20). Even a single-atom contact of Pt sustains multiple conduction channels with intermediate transmissions between 0 and 1 that contribute to the transport properties (Fig. 4B), which is at variance with the Au case. These additional channels originate from the contribution of d-orbitals in Pt (11). Further, our simulations show that the electrical and thermal conductance histograms for Pt are relatively featureless and that there are no strongly preferred conductance values at room temperature (20).

To unambiguously test these predictions, we performed measurements with a Pt-coated scanning probe and a Pt substrate, using the same methodology that we used for Au atom junctions. The measured electrical and thermal conductance traces (Fig. 4C) show plateaus. A histogram-based analysis of 100 Pt traces (Fig. 4D) revealed that the Lorenz ratio is very close to 1 and therefore obeys the Wiedemann-Franz law. However, histograms of electrical and thermal conductance traces do not show conductance quantization (20), in agreement with our computational predictions. This demonstrates that thermal conductance quantization is not a universal feature of all metallic systems at room temperature.

Our work provides insights into thermal transport in atomic-size $\mathrm{Au}$ and Pt contacts and reveals conductance quantization at room temperature in $\mathrm{Au}$ atom junctions. We have also established the applicability of the Wiedemann-Franz law for analyzing thermal transport in metallic atomic-size contacts. The scanning calorimetric probes described here will enable thermal transport studies in molecular junctions, 1D chains of atoms, and individual polymer chains, all of which have been studied theo- retically and computationally for more than half a century $(13,37)$ but have not been probed experimentally because of the lack of experimental tools.

\section{REFERENCES AND NOTES}

1. E. Pop, Nano Res. 3, 147-169 (2010).

2. D. G. Cahill et al., Appl. Phys. Rev. 1, 011305 (2014)

3. J. Crossno et al., Science 351, 1058-1061 (2016).

4. N. Agrait, A. L. Yeyati, J. M. van Ruitenbeek, Phys. Rep. 377 81-279 (2003).

5. J. C. Cuevas, E. Scheer, Molecular Electronics: An Introduction to Theory and Experiment (World Scientific Series in Nanoscience and Nanotechnology, World Scientific, 2010).

6. E. Scheer et al., Nature 394, 154-157 (1998).

7. H. E. van den Brom, J. M. van Ruitenbeek, Phys. Rev. Lett. 82 1526-1529 (1999)

8. P. J. Wheeler, J. N. Russom, K. Evans, N. S. King, D. Natelson, Nano Lett. 10, 1287-1292 (2010).

9. B. Ludoph, J. M. van Ruitenbeek, Phys. Rev. B 59 12290-12293 (1999)

10. P. Reddy, S. Y. Jang, R. A. Segalman, A. Majumdar, Science 315, 1568-1571 (2007)

11. C. Evangeli et al., Nano Lett. 15, 1006-1011 (2015).

12. W. Lee et al., Nature 498, 209-212 (2013).

13. Y. Dubi, M. Di Ventra, Rev. Mod. Phys. 83, 131-155 (2011).

14. K. Schwab, E. A. Henriksen, J. M. Worlock, M. L. Roukes, Nature 404, 974-977 (2000).

15. 0. Chiatti et al., Phys. Rev. Lett. 97, 056601 (2006).

16. M. Meschke, W. Guichard, J. P. Pekola, Nature 444, 187-190 (2006).

17. S. Jezouin et al., Science 342, 601-604 (2013).

18. M. Partanen et al., Nat. Phys. 12, 460-464 (2016)

19. B. Gotsmann, M. A. Lantz, Nat. Mater. 12, 59-65 (2013).

20. Materials and methods are available as supplementary materials.

21. S. Sadat, E. Meyhofer, P. Reddy, Rev. Sci. Instrum. 83, 084902 (2012).

22. S. Sadat, E. Meyhofer, P. Reddy, Appl. Phys. Lett. 102, 163110 (2013).

23. B. Xu, N. J. Tao, Science 301, 1221-1223 (2003)

24. L. Olesen et al., Phys. Rev. Lett. 72, 2251-2254 (1994).

25. J. M. Krans et al., Phys. Rev. Lett. 74, 2146 (1995).

26. L. Olesen et al., Phys. Rev. Lett. 74, 2147 (1995).

27. N. W. Ashcroft, N. D. Mermin, Solid State Physics (Holt, 1976).

28. U. Sivan, Y. Imry, Phys. Rev. B 33, 551-558 (1986).

29. L. Cui et al., Nat. Commun. 8, 14479 (2017).

30. M. Dreher et al., Phys. Rev. B 72, 075435 (2005).

31. C. Schirm et al., Nat. Nanotechnol. 8, 645-648 (2013).

32. F. Pauly et al., New J. Phys. 10, 125019 (2008).

32. F. Pauly et al., New J. Phys. 10, 125019 (2008).
33. M. Bürkle, T. J. Hellmuth, F. Pauly, Y. Asai, Phys. Rev. B 91, 165419 (2015)

34. A. Jain, A. J. H. McGaughey, Phys. Rev. B 93, 081206 (2016). 35. J. P. Bergfield, C. A. Stafford, Nano Lett. 9, 3072-3076 (2009).

36. V. Balachandran, R. Bosisio, G. Benenti, Phys. Rev. B 86 , 035433 (2012).

37. N. Li et al., Rev. Mod. Phys. 84, 1045-1066 (2012).

\section{ACKNOWLEDGMENTS}

P.R. acknowledges support from the U.S. Department of Energy Basic Energy Sciences program (award no. DE-SC0004871). P.R. and E.M. acknowledge support from the U.S. Office of Naval Research (N00014-16-1-2672) and the U.S. National Science Foundation (CBET 1235691). J.C.C. acknowledges funding from the Spanish Ministry of Economy, Industry and Competitiveness (contract no. FIS2014-53488-P) and thanks the German Research Foundation (DFG) and Collaborative Research Center (SFB) 767 for sponsoring his stay at the University of Konstanz as a Mercator Fellow. M.M. and P.N. acknowledge funding from the SFB 767 and computer time granted by the John von Neumann Institute for Computing and the bwHPC framework program of the State of Baden-Württemberg, Germany. J.C.K. and F.P. were financially supported by the Carl Zeiss Foundation, the SFB 767 of the DFG, and the Junior Professorship Program of the Baden-Württemberg Ministry of Science, Research, and the Arts. We acknowledge the Lurie Nanofabrication Facility for facilitating the fabrication of devices. All of the data are available in the main paper and the supplementary materials. 